\title{
Comparative aspects of surgical treatment of the patients with thoracic aorta diseases
}

\author{
Vladimir Dikolayev ${ }^{1}{ }^{2}$, Adilzhan Albazarov ${ }^{2}$, Turlybek Tuganbekov ${ }^{1}{ }^{2}$, Vladimir Grigorevsky ${ }^{1}$, Kulsara \\ Rustemova $^{1}$, Saule Nurakaeva ${ }^{2}$, Murat Raikhanov ${ }^{3}$
}

'Department of Surgical Diseases, Astana Medical University, Nur-Sultan city, Republic of Kazakhstan ${ }^{2}$ Cardiac Surgery Department, National Scientific Medical Research Center, Nur-Sultan city, Republic of Kazakhstan

${ }^{3}$ Cardiac Surgery Department, Regional Cardiac Center», Pavlodar city, Republic of Kazakhstan

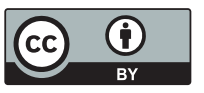

This work is licensed under a Creative Commons Attribution 4.0 International License

Received: 2019-04-30

Accepted: 2018-05-20

UDC: 616.1

\section{J Clin Med Kaz 2019; 2(52):42-49}

Corresponding Author: Vladimir Dikolayev, Cardiac Surgery Department, National Scientific Medical Center. Address: 43/1, Pobeda Street, ap. 1, 010000, Nur-Sultan, Republic of Kazakhstan.

Tel.: 7 (717)2 38-50-61, mob.: +77752249806.

E-mail: dikolayevv@mail.ru

\section{Abstract}

Objective: To carry out comparative analysis of the various surgical methods in patients with thoracic aorta pathology (aneurism, dilatation, dissection) in combination with bicuspid aortic valve in comparison to tricuspid aortic valve patients and to choose most appropriate approach of the surgical treatment in correlation with thoracic aorta sizes.

Material and methods: In this work 212 case records of two patient groups with thoracic aorta pathology were analyzed, operated from 2013 until 2015 in cardiothoracic department of the "National Scientific Medical Center" JSC, to whom 4 surgical approaches were applied: Bentall de Bona procedure, supracoronary aorta replacement procedure, David procedure, Borst procedure.

Results: Usage of Bentall de Bona procedure in patients with bicuspid aortic valve was related to less risk of postoperative complications in comparison with the group of tricuspid aortic valve patients $(17,5 \%$ vs $39,5 \%, \mathrm{p}=0,01)$. Surgical treatment with the usage of David procedure in observation group with BAV eliminates aortic valve insufficiency (degree of aortic valve insufficiency pre and postoperative $-2,3 \pm 1,3$ and $0,9 \pm 1,1$ respectively, $p=0,04$ ).

Conclusion: Surgical treatment of thoracic aorta pathology in patients with bicuspid aortic valve (mainly with stenosis) with the usage of Bentall de Bona approach is accepted as reasonable because it eliminates most considerably the implications of aortopathy and in this regard considered to be the most effective. David procedure is indicated to patients with pathology of thoracic aorta in combination with bicuspid aortic valve (mainly with insufficiency).

Key words: bicuspid aortic valve, thoracic aneurysm, Bentall de Bona procedure, David procedure

\section{ҚОЛҚАНЫҢ КЕУДЕ БӨЛІГІНІН АУРУЛАРЫ БАР ПАЦИЕНТТЕРДІ ХИРУРГИЯЛЫҚ ЕМДЕУДІҢ САЛЫСТЫРМАЛЫ АСПЕКТІЛЕРІ}

Диколаев В.Д.1, ${ }^{2}$, Альбазаров А.Б. ${ }^{2}$, Туганбеков Т.У.1, ${ }^{2}$, Григоревский В.П. ${ }^{1}$, Рустемова К.Р. ${ }^{1}$, Нуракаева С.С. ${ }^{2}$, Райханов М. ${ }^{3}$

'Хирургиялық аурулар бөлімі, Астана медициналық университеті, Нұр-Сұлтан қаласы, Қазақстан Республикасы

${ }^{2}$ Кардиохирургия бөлімі, Ұлттық ғылыми медициналық орталық» АҚ, Нұр-Сұлтан қаласы, Қазақстан Республикасы

${ }^{3}$ Кардиохирургия бөлімі, облыстық кардиологиялық орталық, Павлодар қаласы, Қазақстан Республикасы

\section{ТҰЖЫРЫМДАМА}

Мақсаты: Қолқаның кеуде бөлігінің патологиясы кезінде емдеудің әртүрлі хирургиялық әдістеріне салыстырмалы талдау жүргізу (аневризма, дилятация, қатпарлану) үш жармалы салыстырғанда екі жармалы қолқа қақпақшасы бар емделушілерде қолқаның кеуде бөлігінің өлшемімен корреляцияда операциялық араласудың ең оңтайлы тәсілін таңдау.

Материалдар мен әдістер: Жұмыста 2013-2015 жылдары аралығында «Ұлттық ғылыми медициналық орталық» АҚ кардиоторакалдық бөлімшесінде операция жасалған аортаның кеуде бөлігінің патологиясы бар екі бақылау топтарының ауру тарихтары талданды, оларға 4 жедел әдіс қолданылды: Бенталл де Бон операция, аортаны супракоронарлық протездеу, Дэвид операциясы, Борст операциясы.

Нәтижелер: Екі жармалы аорталық қақпақшасы бар пациенттерде Бенталл де Бонн операциясы үш жармалы аорталық қақпақшасы бар тобымен салыстырғанда отадан кейінгі асқынулардың пайда болуының ең аз қаупімен байланысты (17,5\% vs 39,5\%, p=0,01). ЕЖАҚ бар бақылау тобында Дэвид әдісі бойынша операциялық емдеу аорталық қақпақша функциясы жеткіліксіздігін шынымен жояды (Ақ-дағы операцияға дейінгі және операциядан кейінгі жетіспеушілік дәрежесі - сәйкесінше 2,3 \pm 1,3 және 0,9 \pm 1,1, p=0,04).

қорытынды: Екі жармалы аорталық қақпақшасы (стенозы басым) бар пациенттерде аортаның кеуде бөлігінің патологиясын Бенталл де Бонн әдісі бойынша жедел емдеу ең қолайлы болып табылады, себебі аортопатияның көріністерін барынша радикалды жоюға мүмкіндік береді, осыған байланысты ең тиімдісі болып табылады. Дэвид операция екі жармалы аорталық қақпақшасымен (жетіспеушілігі басым) қатар аортаның кеуде бөлігінің патологиясы бар пациенттерге көрсетілген.

Негізгі сөздер: екі жармалы қолқа қақпақшасы, аортаның кеуде бөлігінің патологиясы, Бенталл де Бон операциясы, Дэвид операциясы 


\section{СРАВНИТЕЛЬНЫЕ АСПЕКТЫ ХИРУРГИЧЕСКОГО ЛЕЧЕНИЯ ПАЦИЕНТОВ С ЗАБОЛЕВАНИЯМИ ГРУДНОГО}

ОТДЕЛА АОРТЫ

Диколаев В.Д. ${ }^{1},{ }^{2}$, Альбазаров А.Б ${ }^{2}$, Туганбеков Т.У.1, ${ }^{2}$, Григоревский В.П.. ${ }^{1}$ Рустемова К.Р. ${ }^{1}$, Нуракаева С.С. ${ }^{2}$, Райханов М. ${ }^{3}$

${ }^{1}$ Отдел кардиохирургических болезней, Медицинский университет Астана, город Нур-Султан, Республика Казахстан

${ }^{2}$ Отдел кардиохирургии, Национальный научный медицинский центр, город Нур-Султан, Республика Казахстан

${ }^{3}$ Отдел кардиохирургии, Областной кардиологический центр, город Павлодар, Республика Казахстан

\section{PEЗЮME}

Цель: Провести сравнительный анализ различных хирургических методов лечения при патологии грудного отдела аорты (аневризма дилятация, расслоение) у пациентов с двустворчатым аортальным клапаном в сравнении с трехстворчатым, выбрать наиболее оптимальный способ оперативного вмешательства в корреляции с размерами грудного отдела аорты.

Материалы и методы: В работе были проанализированы истории болезней двух групп наблюдения (212 пациентов) с патологией грудного отдела аорты, оперированных в кардиоторакальном отделении АО «Национальный научный медицинский центр» в период с 20132015г., которым были применены 4 оперативных методики: операция Бенталла де Бона, супракоронарное протезирование аорты, операция Дэвида, операция Борста.

Результаты: Операция Бенталла де Бона у пациентов с двустворчатым аортальным клапаном связана с меньшим риском возникновения послеоперационных осложнений в сравнении с группой с трехстворчатым аортальным клапаном (17,5\% vs 39,5\%, p=0,01). Оперативное лечение по методу Дэвид в группе наблюдения с ДАК достоверно устраняет недостаточность на аортальном клапане (степень недостаточности на АК до и после операции - 2,3 $\pm 1,3$ и 0,9 $\pm 1,1$ соответственно, $p=0,04)$.

Выводы: Оперативное лечение патологии грудного отдела аорты у пациентов с двустворчатым аортальным клапаном (преимущественно со стенозом) по методу Бенталла де Бона является наиболее приемлемым, так как позволяет максимально радикально устранить проявления аортопатии, и в связи с этим является наиболее эффективным. Операция Дэвида показана пациентам с патологией грудного отдела аорты в сочетании с двустворчатым аортальным клапаном (преимущественно с недостаточностью). Дэвида

Ключевые слова: двустворчатый аортальный клапан, аневризма грудного отдела аорты, операция Бенталла де Бона, операция

\section{Введение}

Двустворчатый аортальный клапан - наиболее часто встречающийся врожденный порок сердца. По данным литературных источников частота встречаемости двустворчатого аортального клапана в общей популяции $2 \%$ (в 2-3 раза чаще у мужчин) [1]. В последние десятилетия структура клапанной болезни существенно изменилась. Снижение частоты ревматизма привело к значительному возрастанию роли врожденных аномалий и в первую очередь двустворчатого аортального клапана. Немалая доля людей с этим пороком доживает до старости без какихлибо серьезных проблем со стороны аортального клапана, однако в ряде случаев вследствие различных процессов формируется гемодинамически значимый порок этого клапана. Пациенты с таким пороком имеют определенные клинические проявления, нарушения кровотока в области аортального клапана относительно легко диагностируются и, как правило, больные вовремя направляются на хирургическое лечение. По данным мировой статистики частота осложненных форм течения двустворчатого аортального клапана варьирует в пределах от $35-40 \%$ до $75 \%[2,3]$.

Другим состоянием, ассоциированным с наличием двустворчатого аортального клапана, является расширение восходящего отдела аорты. В настоящее время дилятация аорты с последующим разрывом или расслоением представляет одну из главных проблем в кардиохирургии [4].

Исследованиями было доказано, что размер аорты продолжает расти после протезирования двустворчатого аортального клапана [5]. В связи с этим в данной группе пациентов наиболее часто наблюдаются случаи повторных оперативных вмешательств по причине развития аневризмы, а в отдаленном периоде риск возникновения расслоения и внезапной смерти [6,7]. Современными исследованиями по данным компьютерной и магнитно-резонансной томографии с контрастированием магистральных сосудов был выявлен диффузный характер аортопатии, который распространяется от корня до дуги грудной аорты $[8,9]$. В настоящее время существуют дебаты относительно, является ли данное патологическое состояние следствием изменений во внутренней структуре стенки аорты либо существенную роль играет гемодинамический фактор [10].

За прошедшее время с целью хирургического лечения пациентовсдвустворчатымаортальнымклапаномвсочетании с аневризмой аорты было предложено множество методик. В основе данных методик преобладает протезирование восходящего отдела грудной аорты синтетическим протезом. Некоторые исследователи предлагают радикальный подход в определении границ резекции, мотивируя это тем, что в отдаленном периоде после оперативного вмешательства существует риск возникновения дилятации и расслоения в оставшейся дистальной части и в корне аорты $[11,12,13]$.

Таким образом, несмотря на наличие множества научных работ посвященной теме хирургического лечения пациентов с двустворчатым аортальным клапаном в сочетании с заболеваниями грудной аорты, остается актуальной проблема неопределенности и субъективизма выбора хирургического метода лечения, определения границ резекции и показаний к оперативному лечению. Это обстоятельство обусловило выполнение данного исследования, целью которого явилось изучение эффективности проведенных вмешательств на основе ретроспективного анализа более чем двухсот случаев хирургического лечения патологии грудного отдела аорты.

\section{Материалы и методы}

Для исследования были отобраны из архива АО «Национальный научный медицинский центр» (далее $\mathrm{AO}$ «ННМЦ») истории болезни 212 пациентов с патологией грудного отдела аорты (дилятация, аневризма, расслоение) в возрасте от 20 - 76 лет (средний возраст 55,98 \pm 12 ), находившихся на лечении в отделении кардиоторакальной хирургии АО «ННМЦ» в период с 2013-2015 гг. Учитывая сравнительный характер исследования, все пациенты были разделены на 2 группы наблюдения по характеру анатомического строения аортального клапана. Первая группа - 101 пациент с двустворчатым аортальным клапаном (ДАК). Вторая группа - 111 пациентов с трехстворчатым аортальным клапаном (ТАК).

Критерии включения:

1. Пациенты, у которых в дооперационном периоде 
по данным ЭхоКГ был диагностирован ДАК, ТАК в сочетании с аневризматическим расширением, дилятацией, расслоением грудного отдела аорты

2. Пациенты, у которых ДАК, ТАК был диагностирован в интраоперационном периоде макроскопически вместе с патологией грудного отдела аорты.

Критерии исключения:

1. Отсутствие данных в истории болезни пациентов об анатомическом строении аортального клапана.

2. Пациенты с одностворчатым, четырехстворчатым аортальным клапаном.

3. Пациенты с ДАК, ТАК в сочетании с патологией грудного отдела аорты, отказавшиеся от хирургического вмешательства.

4. Пациенты с ДАК, ТАК без патологии грудного отдела аорты.

По данным антропометрии пациентов отмечено, что в группе наблюдения с ДАК $(75,24 \%)$ в сравнении с пациентами с ТАК (62,16\%) преобладают пациенты с мужским полом $(\mathrm{p}=0,04)$. Средний возраст пациентов с ДАК $(52,01 \pm 13,54)$ был ниже в сравнении с группой наблюдения с ТAK $(59,60 \pm 8,93)$.

По исходным клиническим данным по наличию у пациентов сопутствующих заболеваний отмечено, что в группе с ТАК доля сопуствующих заболеваний была выше в сравнении с группой с ДАК. Статистически значимые различия были выявлены по критерию артериальная гипертензия, ИБС $(\mathrm{p}=0,04)$. Функциональный класс хронической сердечной недостаточности $(\mathrm{XCH})$ оценивался согласно классификации Нью-Йоркской ассоциации кардиологов (NYHA). Подавляющее большинство пациентов в обеих группах до операции находились во II функциональном классе по NYHA.

\section{Методы исследования:}

1. Трансторакальная эхокардиография (ЭхоКГ).

2. Рентгенография органов грудной клетки в прямой и левой боковой проекции.

3. Электрокардиограмма с 12 отведениями.

4. Компьютерная томография грудного отдела аорты с контрастированием.

5. Статистический метод.

\section{Трансторакальная ЭхоКГ}

Основным инструментальным методом диагностики заболеваний грудного отдела аорты и морфологии аортального клапана (АК) была трансторакальная ЭхоКГ. ЭхоКГ проводилась всем пациентам в до и послеоперационном периоде и явилась основным методом, для которого был применен статистический анализ количественных параметров. Исследование проводилось на аппарате «PhillipsEpiQ 7». Данный метод позволял визуализировать корень аорты, восходящую аорту, клапанный аппарат сердца, размеры полостей, градиенты давления.

Положение пациента лежа на спине, голова слегка приподнята. При неудовлетворительных результатах больного укладывали на левый бок. Регистрацию эхокардиограмм производили, как правило, в пяти стандартных направлениях ультразвукового луча из парастернального доступа. При необходимости регистрацию могли производить из апикального, правого парастернального и эпигастрального доступа. Основные ЭхоКГ параметры пациентов обеих групп отражены в Таблице 1.

Таблица 1 ЭхоКГ параметры групп наблюдения до оперативного вмешательства

\begin{tabular}{|l|l|l|l|l|}
\hline № & Показатель & Среднее ТАК & Среднее ДАК & t-критерий \\
\hline 1 & ФВЛЖ, 0 & 53,0541 & 53,3663 & $-0,2030$ \\
\hline 2 & Конечно-диаст. объем ЛЖ, мл & 153,2268 & 158,6733 & $-0,5606$ \\
\hline 3 & Конечно-диаст. размер ЛЖ, мм & 55,7387 & 55,0198 & 0,5356 \\
\hline 4 & Размер ЛПС, мм & 43,6757 & 40,6634 & 2,7931 \\
\hline 5 & Размер основания аорты, мм & 30,6306 & 30,7822 & $-0,2520$ \\
\hline 6 & Размер синусов Вальсальвы, мм & 42,9730 & 39,8416 & 2,5658 \\
\hline 7 & Размер восходящего отдела аорты, мм & 49,5946 & 48,2475 & 1,1947 \\
\hline 8 & Степень недостаточности МК & 0,6667 & 0,4257 & 1,6934 \\
\hline 9 & Средний градиент на МК & 0,5407 & 0,3327 & 0,801 \\
\hline 10 & Степень недостаточности на АК & 2,1991 & 2,1931 & 0,01 \\
\hline 11 & Средний градиент на АК & 24,3446 & 0,09 \\
\hline
\end{tabular}

Систолическая функция левого желудочка в обеих группах наблюдения статистически значимо не различалась. Показатель фракции выброса (ФВ) левого желудочка для групп наблюдения с ДАК и ТАК составил $(53,4 \pm 12,2 \%, 53,1 \pm 10,2 \%)$. Объемные размеры левого желудочка также значимо статистически не различались и составили соответственно для пациентов с ДАК (КДО ЛЖ$158,7 \pm 72$ мл, КДР ЛЖ - 55,0 $\pm 11,3$ мм), для пациентов с ТАК

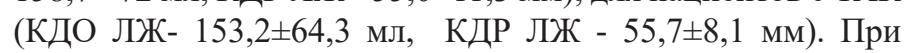
проведении трансторакальной ЭхоКГ в дооперационном периоде особую значимость в нашем исследовании играли 2 размера грудного отдела аорты:

А. Максимальный диаметр на уровне синусов Вальсальвы.

В. Максимальный диаметр на уровне тубулярной части восходящей аорты.

Согласно мировым рекомендациям эти два показателя используются для определения границ резекции аорты и, соответственно, установления показаний для выбора оперативного вмешательства. У 61,4\% пациентов с ДАК несмотря на отсутствие расширения синуса Вальсальвы, тем не менее применялась методика Бентала де Бона, исходя из соображений предотвращения формирования аневризмы корня аорты.

Для пациентов с ДАК средний размер со стандартным отклонением для синусов Вальсальвы и ВОА составлял 39,9 $\pm 0,9$ мм и 48,3 $\pm 0,8$ мм. Для пациентов с ТАК средний размер со стандартным отклонением для синусов Вальсальвы и ВОА составлял 42,9 \pm 0,9 мм и 49,6 $\pm 0,8$ мм, соответственно. При этом по показателю размер синусов Вальсальвы было 
выявлено статистически значимое различие $(\mathrm{p}=0,01)$.

Статистически значимое различие также было отмечено по критерию: средний градиент на аортальном клапане $(\mathrm{p}=0,01)$. В группе с ДАК отмечается более высокий градиент на аортальном клапане $(24,4 \pm 23)$ в сравнении с группой ТАК $(12,9 \pm 17,2)$.

\section{Рентгенография органов грудной клетки}

Всем пациентам без исключения проводилась рентгенография органов грудной клетки в прямой и левой боковой проекции. При проведении рентгенографического исследования анализировались размеры сердца, форма сердечной тени, наличие и выраженность признаков легочной гипертензии.

\section{Электрокардиографическое исследование}

Всем больным выполнялась 12-канальное электрокардиографическое исследование (ЭКГ). Анализировались ритм, нарушения проводимости.

По результатам анализа ЭКГ отмечено, что в группе наблюдения с ТАК в дооперационном периоде больше наблюдаются случаев фибрилляции предсердий ФП (24,3\%), блокад ножек пучка Гиса $(15,3 \%)$ в сравнении с группой наблюдения с ДАК (16\%, 9,9\%).

Мультиспиральная компьютерная томография с контрастированием

Для уточнения размеров аорты, распространенности аневризмы, исключения расслоения ряду пациентов перед операцией выполнена мультиспиральная компьютерная томография (МСКТ) с контрастированием. Также МСКТ выполнялась пациентам при повторных вмешательствах с целью оценки прилежания структур сердца к передней грудной стенке.

\section{Статистический метод}

Для статистического сравнения основной и контрольной группы применялся параметрический метод Стьюдента (t-критерий Стьюдента), а также методы дисперсионного анализа и непараметрические методы.

Статистический анализ осуществлялся с помощью специализированных программных пакетов STATISTICA 6.1 forWindows.

\section{Методы хирургического лечения}

Все методы хирургического лечения на грудной отдел аорты были условно распределены на 4 группы:

1) Операция супракоронарного протезирования восходящего отдела аорты с раздельным протезированием, пластикой аортального клапана.

По данной методике оперировано 64 (30,1\%) больных, из них 13 (6,1\%) пациентов были с ДАК, 51 (24\%) с ТАК.

2) Операция протезирования корня аорты с использованием клапансодержащего кондуита (операция Бенталла де Бона).

По данной методике прооперировано 118 (55,6\%) пациентов, из них 80 (37,7\%) с ДАК, 15 (17,9\%) пациентов c TAK.

3) Клапаносохраняющая операция Дэвида с протезированием корня аорты.

По данной методике прооперировано 22 (10,7\%) пациентов, из них 7 (3,3\%) с ДАК, 38 (7,07\%) пациентов с TAK.

4) Оперативное вмешательство на грудной отдел аорты с протезированием дуги аорты (операция Борста).

По данной методике прооперировано 8 (3,7\%) пациентов, из них 1 (0,47\%) с ДАК, 7 (3,30\%) пациентов с TAK.

\section{Результаты}

При проведении анализа результатов хирургического лечения был применен статистический метод обработки данных пациентов для двух групп наблюдения. Все методы хирургических вмешательств, которые были применены у пациентов были закодированы в 4 группы (Таблица 2).

Таблица 2 Таблица расшифровки кодов операции видов вмешательства

\begin{tabular}{|l|l|}
\hline Код операции & Наименование операция, вида вмешательства \\
\hline 1 & Бенталла де Бона \\
\hline 2 & Супракоронарное протезирование аорты \\
\hline 3 & Дэвида \\
\hline 4 & Борста \\
\hline
\end{tabular}

\section{Сравнительный анализ выбора метода хирургического лечения у больных с ДАК и TAK}

С целью ретроспективного анализа размеров грудного отдела аорты по данным ЭхоКГ, выбора метода операции и репрезентативности выборки, исходные данные были обработаны в отношении среднего размера синусов Вальсальвы и восходящего отдела аорты

Для группы наблюдения с ДАК были получены следующие результаты (Рисунок 1,2).

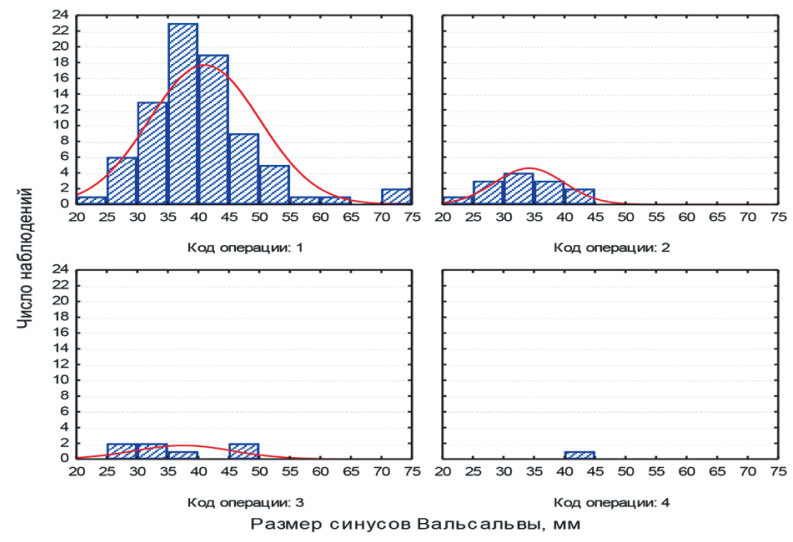

Рисунок 1 - Категориальная гистограмма по показателю «Размер синусов Вальсальвы» и разреза кода операции вида вмешательства у пациентов с ДАК.

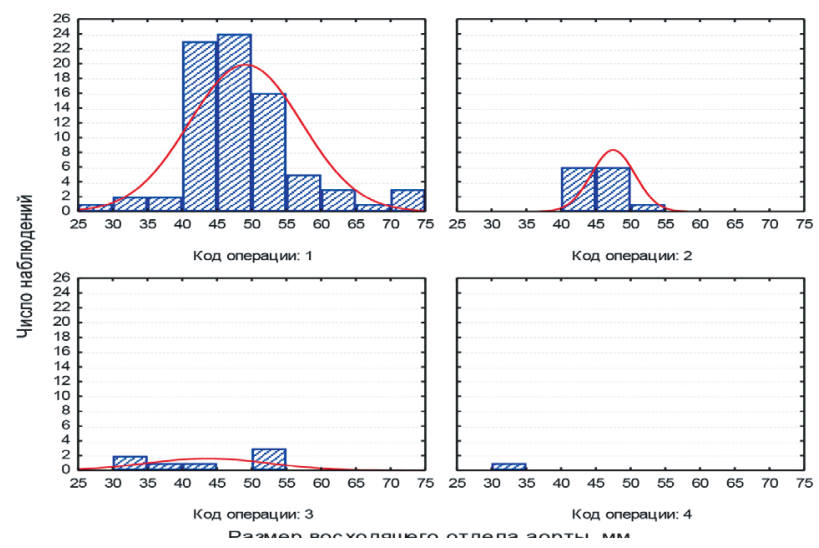

Рисунок 2 - Категориальная гистограмма по показателю «Размер восходящего отдела аорты» в разрезе кода операции - вида вмешательства у пациентов с ДАК. 
Анализируя полученые данные для группы наблюдения с ДАК было выявлено, что в 79,2\% случаев применялся метод хирургического лечения - операция Бенталла де Бона, несмотря на то, что средний размер грудного отдела аорты до операции для синусов Вальсальвы и ВОА составлял $39,8 \pm 0,9$ мм и 48,3 $\pm 0,8$ мм соответственно, т.е. средний размер синусов Вальсальвы не являлся показанием к протезированию корня аорты. Данное наблюдение доказывает, что размер грудного отдела аорты у пациентов с ДАК не играет лимитирующую роль в вопросе выбора метода хирургического лечения.

Соответствующим образом были обработаны данные для группы пациентов с ТАК (Рисунок 3,4$)$.

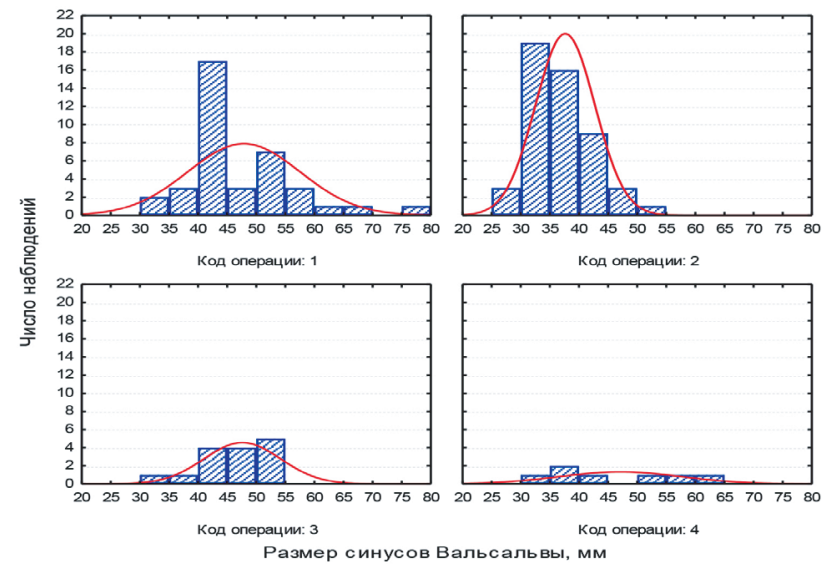

Рисунок 3 - Категориальная гистограмма по показателю « Размер синсуов Вальсальвы» в разрезе кода операции- вида вмешательства у пациентов с ТАК.

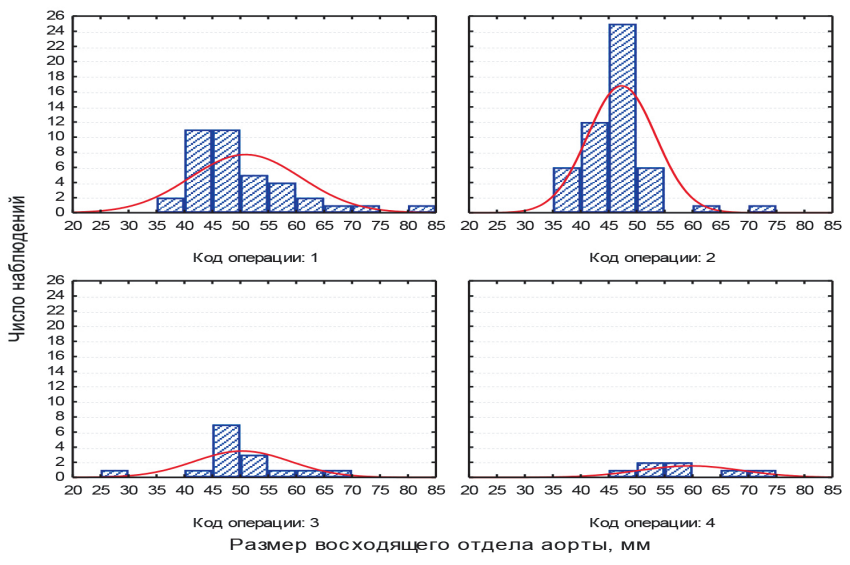

Рисунок 4 - Категориальная гистограмма по показателю « размер восходящего отдела аорты» в разрезе кода операции - вида вмешательства у пациентов с ТАК.

Анализируя данные группы наблюдения с ТАК, было выявлено, что супракоронарноепротезирование восходящего отдела аорты было наиболее часто применяемой техникой $(45,9 \%)$ из четырех методов лечения. Хирургическое лечение по методике Бенталла де Бона наблюдалось в 34,2\%.

Также было отмечено, что средний размер грудного отдела аорты со стандартным отклонением для синусов Вальсальвы и ВОА составлял 43,0 \pm 0,9 мм и 49,6 \pm 0,8 мм. Несмотря на то, что средний размер синусов Вальсальвы составлял 43,0 \pm 0,9 мм, каждый третий пациент был прооперирован по методике Бенталла де Бона, что является доказательство субъективного подхода в выборе оперативного вмешательства.

\section{Анализ эффективности методов хирурги- ческого лечения}

С целью определения наиболее эффективного метода хирургического лечения был произведен статистический анализ данных пациентов с ДАК в разрезе зависимости исхода и послеоперационных осложнений от примененного вида операции у пациентов. Учитывая статистический анализ данных, критерии послеоперационного осложнения и исход пациентов после хирургического вмешательства были закодированы числовыми символами (исход: 1-выздоровление, 0-смерть; послеоперационные осложнения: 1- наличие осложнений, 0- отсутствие осложнений) (Рисунок 5).

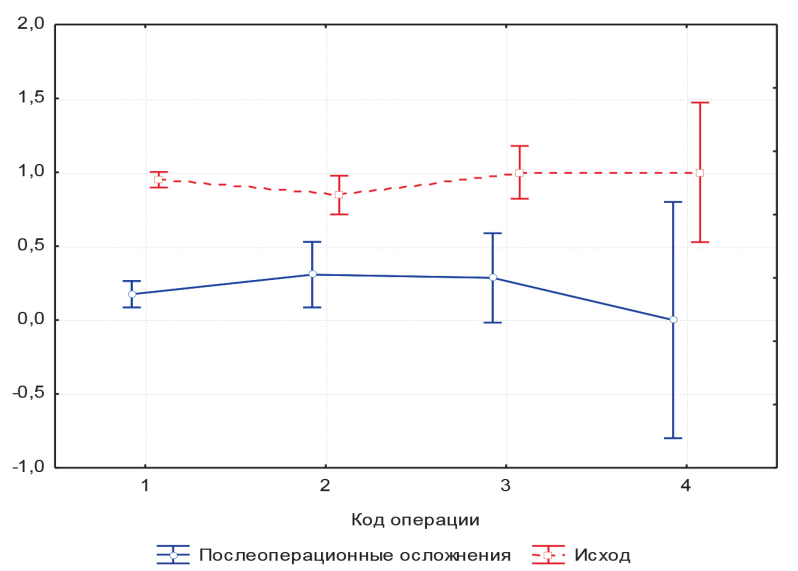

Вертикальные столбцы равны $\pm 0,95$ доверительных интервалов для среднего.

Рисунок 5 - График соответствия (зависимости) послеоперационных осложнений и исходов операции по результатам факторного анализа.

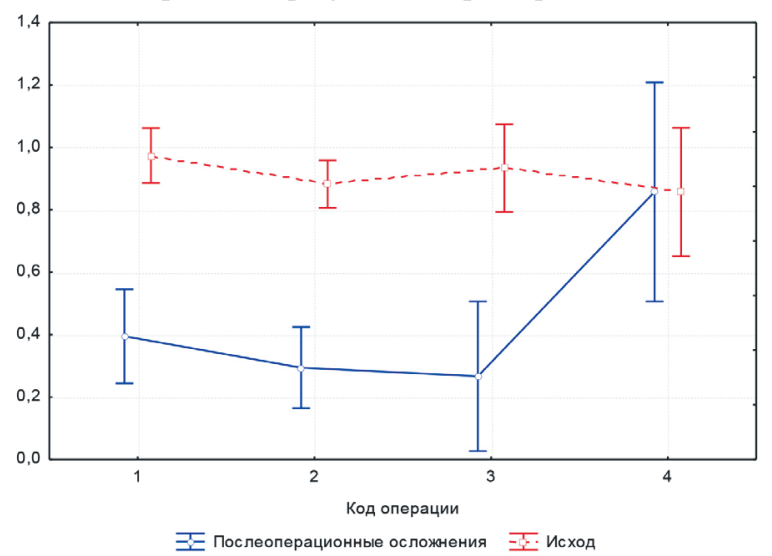

Вертикальные столбцы равны $\pm 0,95$ доверительных интервалов для среднего

Рисунок 6 - График соответствия (зависимости) послеоперационных осложнений и исходов операций по результатам факторного анализа.

Из результатов однофакторного анализа следует, что нет статистически достоверной связи между выбором хирургического лечения и исходом операции (количество послеоперационных осложнений, смерть или выздоровление). Соответственно все 4 метода могут быть применены с удовлетворительными непосредственными результатами для пациентов с ДАК. 
По результатам статистического анализа группы наблюдения с ТАК следует, что статистически значимое влияние проявление эффектов влияния факторов на наблюдаемые показатели проявляются между фактором “Операция” (Код операции) и регистрируемым показателем “Послеоперационные осложнения”. Влияния фактора “Операция” и проявление эффекта на показатель “Исход” не наблюдается (Рисунок 6).

Исходя из результатов статистического анализа данных для пациентов с ТАК выбор метода хирургического лечения оказал существенноевлияние на частотупослеоперационных осложнений. При этом у 6,3\% пациентов в группе с ТАК с расслаивающей аневризмой аорты, применялся метод Борста, требующий циркуляторного ареста с гипотермией до $18-20^{\circ} \mathrm{C}$, что в значительной мере объясняет количество выявленных послеоперационных осложнений в данной группе. Метод супракоронарного протезирования аорты с учетом того, что данный метод был наиболее применяемый у пациентов с ТАК, оказался наиболее эффективным методом хирургического лечения в отношении риска возникновения послеоперационных осложнений.

\section{Сравнительный анализ эффективности клапаносохраняющей операции (операция Дэвид)}

Для определения эффективности клапансохраняющей операции (операция Дэвид) были проанализированы исходные данные 2-х групп наблюдения. Анализу подверглись исходные ЭхоКГ данные до операции и в раннем послеоперационном периоде.

Статистически значимый положительный результат хирургического лечения в группе пациентов с ДАК был выявлен по такому показателю как: степень недостаточности на аортальном клапане (степень недостаточности на АК до и после операции - 2,286 $\pm 1,254$ и $0,857 \pm 1,069$ соответственно, $\mathrm{p}=0,0353)$. Несмотря на сохранение собственного дефектного клапана, операция Дэвида позволяет скорригировать геометрию корня аорты, что ведет к уменьшению недостаточности АК. Это, в свою очередь, позволяет устранить потребность в пожизненном принятии антикоагулянтов непрямого действия.

В группе наблюдения с ТАК дополнительно к вышеуказанному показателю значимые результаты были выявлены в показателях: конечно-диастолический размер ЛЖ, конечно-диастолический объем ЛЖ. В этой группе операция Дэвида также позволила сохранить нативный клапан с помощью коррекции геометрии корня аорты, в результате чего была уменьшена недостаточность на АК и снизились объемные показатели ЛЖ (КДО,КДР) по данным послеоперационных ЭхоКГ.

\section{Сравнительный анализ непосредственных результатов хирургического лечения}

Методом статистического анализа были проанализированы непосредственные результаты хирургического лечения в обеих группах.

По результатам обработки отмечается, что показатель времени искусственного кровообращения был меньше в группе наблюдения с ДАК в сравнении с группой наблюдения с ТАК $(133,2 \pm 50,9,143,7 \pm 66,9)$. Также в критерии времени ишемии миокарда в группе наблюдения с ДАК отмечен меньший показатель в сравнении с группой наблюдения с ТАК $(91,6 \pm 29,4,101,3 \pm 47,9)$. Оба показателя повлияли на непосредственные результаты хирургического лечения в группах наблюдения в виде снижения количества послеоперационных осложнений.

Количество наблюдений послеоперационных осложнений в группе наблюдения с ДАК (20, т.е. 19,8\%) было меньше в сравнении с группой наблюдения с ТАК (40, т.е. $36 \%$ ) и имело статическую значимую разницу $(\mathrm{p}=0,01)$.

Послеоперационная летальность в группе наблюдения с ДАК составила $5,9 \%$, в то время как в группе наблюдения пациентов с ТАК - 8,1\%. Среди причин летальности в обеих группах наблюдения преобладают такие причины, как острая сердечно-сосудистая недостаточность, сепсис и синдром полиорганной недостаточности.

С целью определения влияния методов хирургического лечения на исход и послеоперационные осложнения были построены диаграммы для двух групп наблюдения.

В группе наблюдения с ДАК оперативное лечение по методу Бенталла де Бона в сравнении с методом супракоронарного протезирования имело лучшие непосредственные результаты в отношении развития риска послеоперационных осложнений и летального исхода $(\mathrm{p}=0,26, \mathrm{p}=0,16)$ (Рисунок 7).

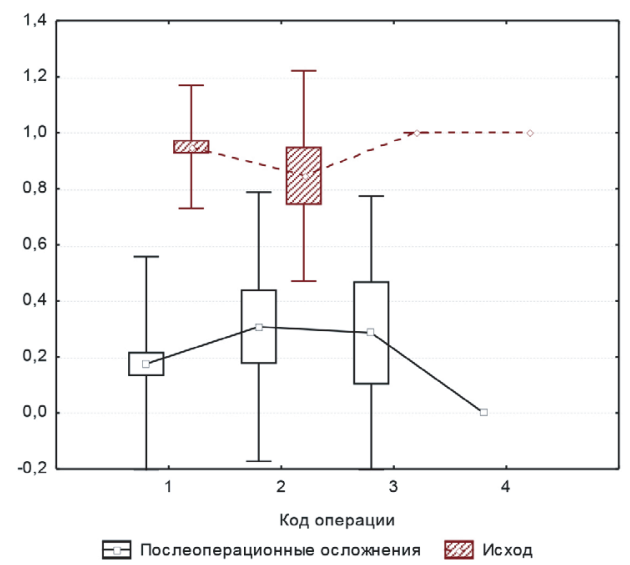

Рисунок 7 - Диаграмма размахов для средних послеоперационных осложнений и исходов в разрезе видов операции для группы наблюдения с ДАК.

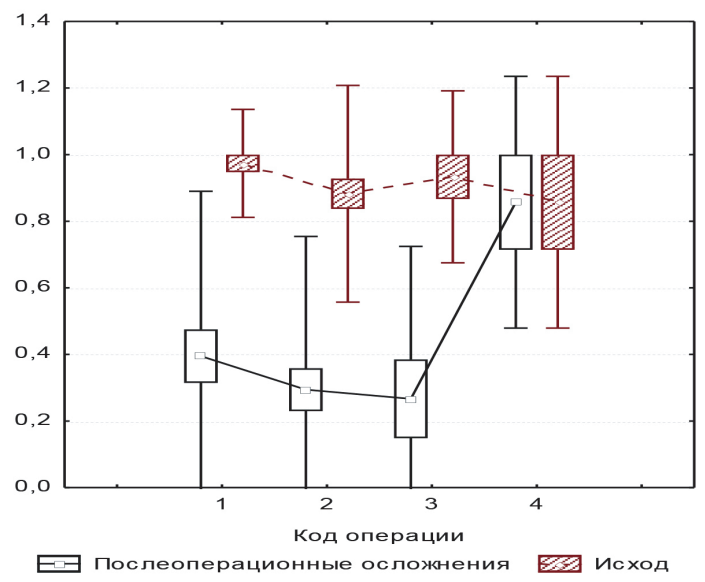

Рисунок 8 - Диаграмма размахов для средних послеоперационных осложнений и исходов в разрезе видов операции для группы наблюдения с ТАК. 
В группе наблюдения с ТАК оперативное лечение по методу Бенталла де Бона имело лучший результат в отношении риска летального исхода в сравнении с методом супракоронарного протезирования $(\mathrm{p}=0,12)$. Несмотря на это по критерию риска возникновения послеоперационных осложнений оперативное лечение супракоронарного протезирования в сравнении с методом Бенталла де Бона показало более низкий показатель $(\mathrm{p}=0,32)$ (Рисунок 8).

С целью определения эффективности каждого метода оперативного лечения между наблюдаемыми группами наблюдения был произведен непараметрический статистический анализ ранговым дисперсионным анализом Краскела-Уоллиса.

По результатам анализа было выявлено, что послеоперационные осложнения у пациентов, прооперированных по методу Бенталла де Бона в группе наблюдения с ТАК (39,5\%) в сравнении с ДАК $(17,5 \%)$ имели статистически значимую достоверную разницу $(\mathrm{p}=0,01)$. По другим видам оперативного вмешательства значимой разницы не было выявлено.

Результат проведенного статистического анализа выявил что, оперативное лечение по методике Бенталла де Бона имело статистически значимый лучший непосредственный результат в раннем послеоперационном периоде в отношении риска возникновения послеоперационных осложнений в сравнении с группой наблюдения с ТАК $(\mathrm{p}=0,01)$. Данный факт определяет эффективность применения данного метода в группе наблюдения с ДАК.

\section{Заключение}

Резюмируя результаты собственных исследований и критически оценивая литературные данные, можно прийти к заключению о том, что хирургическое лечение аневризм аорты в сочетании с ДАК имеет удовлеворительные непосредственные результаты (послеоперационная летальность составила 5,9\%, что соответствует среднестатистическим мировым показателям смертности после вмешательств на аорте). При этом, несмотря на наличие современных рекомендаций, в большинстве случаев выбор метода хирургического лечения и определение показаний к оперативному лечению зависит от индивидуальных предпочтений оперирующего хирурга в каждой конкретной клинической ситуации либо от политики медицинского учреждения. Данное несоответствие связано с недостатком на современном этапе дополнительных критериев (оценка влияния патологического потока на стенку аорты, генетические критерии и т.д.) для стратификации у пациентов риска возникновения жизнеугрожающих состояний, таких как разрыв либо расслоение аорты. Несмотря на это, по результатам наших исследований оперативное лечение аневризм грудного отдела аорты в сочетании с ДАК было проведено согласно рекомендациям Американской Ассоциации Кардиологов (2010 г.), а метод операции Бенталла де Бона у пациентов в группе наблюдения с ДАК является наиболее приемлемым с точки зрения риска возникновения послеоперационных осложнений и патофизиологии аортопатии $(17,50 \%, \mathrm{p}=0,01)$.

Анализ клапаносохраняющего оперативного вмешательства по методу Дэвид у пациентов с ДАК также показал положительные результаты (снижение степени недостаточности на АК $2,3 \pm 1,3$ vs $0,9 \pm 1,1 \mathrm{p}=0,04)$. По результатам литературного обзора и наших наблюдений было определено, что наиболее подходящими кандидатами для данного вида хирургического лечения являются пациенты с аневризмой аорты без грубых морфологических изменений створок АК, преимущественно с недостаточностью (II-ІҮстепень). При этом как показали исследованния, удовлетворительные результаты (10 летняя свобода от повторных хирургических вмешательств по поводу недостаточности на АК) в послеоперационном периоде наблюдаются у пациентов без значимой дилятации аортовентрикулярного соединения, не превышающего 28 мм. В дальнейшем, вероятно, будет необходимо проведение проспективных исследований с целью выявления влияния на долгосрочные результаты хирургического лечения типа ДАК и пространственной ориентации створок. Результаты данных исследований в последующем окажут влияние на определение более узких показаний к клапаносохраняющему оперативному лечению в каждой конкретной клинической ситуации.

\section{Выводы}

По результатам сравнительного анализа выявлено что, наиболее часто в группе наблюдения пациентов с двустворчатым аортальным клапаном (79,2\%) применялся метод оперативного лечения Бенталла де Бона, в то время как в группе наблюдения с трехстворчатым аортальным клапаном метод супракоронарного протезирования восходящего отдела аорты $(45,9 \%)$. При этом операция Бенталла де Бона у пациентов с двустворчатым аортальным клапаном связана с меньшим риском возникновения послеоперационных осложнений в сравнении с группой с трехстворчатым аортальным клапаном (17,5\% vs $39,5 \%$, $\mathrm{p}=0,01)$.

Оперативное лечение по методу Дэвид в группе наблюдения с сохранением двустворчатого аортального клапана достоверно уменьшает недостаточность на аортальном клапане (степень недостаточности на аортальном клапане до и после операции - 2,3 $\pm 1,3$ и 0,9 \pm $1,1$ соответственно, $\mathrm{p}=0,04)$.

По данным проведенного исследования показатели размеров синуса Вальсальвы и восходящего отдела аорты не являются определяющими факторами в решении вопроса о границах резекции грудного отдела аорты и выбора метода операции (в 61,4\% случаев использовалась методика Бенталла де Бона в группе наблюдения с ДАК).

Disclosures: There is no conflict of interest for all authors. 


\section{Литература}

1. Belokon N.A., Pozolkov V.P. Vrozhdennye poroki serdtsa (Congenital heart defects) [in Russian]. Medicine. 1991; 352.

2. Mills P., Leech G., Davies M., et al. The natural history of a non-stenotic bicuspid aortic valve. Br Heart J. 1978; 40:951-957. https:// doi.org/10.1136/hrt.40.9.951

3. Fenoglio, J.J., McAllister, H.A., DeCastro, C.M., Davia, J.E., Cheitlin, M.D. Congenital bicuspid aortic valve after age 20. American Journal of Cardiology. 1977; 39:164-169. https://doi.org/10.1016/S0002-9149(77)80186-0

4. Vallely MP, Semsarian C, Bannon PG. Management of the ascending aorta in patients with bicuspid aortic valve disease. HeartLungCirc. 2008; 17:357-63. https://doi.org/10.1016/j.hlc.2008.01.007

5. Yasuda H, Nakatani S, Stugaard M, et al. Failure to prevent progressive dilation of ascending aorta by aortic valve replacement in patients with bicuspid aortic valve: Comparison with tricuspid aortic valve. Circulation. 2003; 108(11):II291- 4. https://doi.org/10.1161/01. cir.0000087449.03964.fb

6. Russo CF, Mazzetti S, Garatti A, et al. Aortic complications after bicuspid aortic valve replacement: Long-term results. AnnThoracSurg. 2002; 74:S1773- 6; discussion S1792-9. https://doi.org/10.1016/S0003-4975(02)04261-3

7. Borger MA, Preston M, Ivanov J, et al. Should the ascending aorta be replaced more frequently in patients with bicuspid aortic valve disease? J ThoracCardiovascSurg. 2004; 128:677-83. https://doi.org/10.1016/j.jtcvs.2004.07.009

8. Fazel SS, Mallidi HR, Lee RS, et al. The aortopathy of bicuspid aortic valve disease has distinctive patterns and usually involves the transverse aortic arch. $J$ ThoracCardiovascSurg. 2008; 135:901-7, 907.e1-2. https://doi.org/10.1016/j.jtcvs.2008.01.022

9. Westhoff-Bleck M, Meyer GP, Lotz J, et al. Dilatation of the entire thoracic aorta in patients with bicuspid aortic valve: A magnetic resonance angiography study. Vasa. 2005; 34:181-5. https://doi.org/10.1024/0301-1526.34.3.181

10. Guntheroth WG, Spiers PS. Does aortic root dilatation with bicuspid aortic valves occur as a primary tissue abnormality or as a relatively benign poststenotic phenomenon? Am J Cardiol. 2005; 95:820. https://doi.org/10.1016/j.amjcard.2004.12.004

11. Etz CD, Homann TM, Silovitz D, et al. Long-term survival after the Bentall procedure in 206 patients with bicuspid aortic valve. AnnThoracSurg. 2007; 84:1186-93; discussion 1193-4. https://doi.org/10.1016/j.athoracsur.2007.03.057

12. Houel R, Soustelle C, Kirsch M, Hillion ML, Renaut C, Loisance DY. Long-term results of the bentall operation versus separate replacement of the ascending aorta and aortic valve. J HeartValveDis. 2002; 11:485-91.

13. Hagl C, Strauch JT, Spielvogel D, et al. Is the Bentall procedure for ascending aorta or aortic valve replacement the best approach for long-term event-free survival? AnnThoracSurg. 2003; 76:698-703; discussion 703. https://doi.org/10.1016/S0003-4975(03)00568-X

How to cite this article: Vladimir Dikolayev, Adilzhan Albazarov, Turlybek Tuganbekov, Vladimir Grigorevsky, Kulsara Rustemova, Saule Nurakaeva, Murat Raikhanov. Comparative aspects of surgical treatment of the patients with thoracic aorta diseases [in Russian]. J Clin Med Kaz. 2019; 2(52):42-49 